\title{
Control Strategy Research of Vehicle Stability Control System
}

\author{
Youbing Zhu \\ Chongqing College of Electronic Engineering, Chongqing, 401331, China \\ Email:happylover618@126.com
}

\begin{abstract}
Keywords: Vehicle stability control; vehicle dynamics control; control strategy; limit non-linear conditions

Abstract. Vehicle stability control system is also called electronic stability program (ESP) via real-time adjustment of the operating state of the vehicle, it can significantly improve the car and the steering stability in extreme conditions of handling performance. ESP as an automotive active safety technology representatives it has caused wide concern in the world's major automobile manufacturers and research departments. This paper summarizes the results of previous studies based on the first vehicle stability control principle is analyzed, mainly explained why to choose yaw rate and sideslip angle as a control variable; then two control simulation platform policy detailed simulation analysis and comparison in an open loop, and verified by simulation of a variety of conditions, and slip angle estimation and explored based implementations wheel slip rate control.
\end{abstract}

\section{Introduction}

In recent years, with the great development of the economy and vehicle technology, road traffic exhibits with high-speed, traffic-intensive and non-professional driver of the trend, but along with road accidents directly caused enormous loss of human life and property this has become a worldwide social problem [1, 2]. Deaths from road accidents worldwide each year more than 500,000, the number more than 12 million injuries, property damage over 50 billion US dollars, while China is one of the countries most affected by traffic accidents, the grim reality so that people have to pay attention to cars safe question [3].

On dynamics of a vehicle, in an emergency or high-speed sharp turns to avoid the obstacle, the car will enter the large lateral acceleration and sideslip angle limit conditions, the driver can easily produce uncontrollable skid [4-6]. Meanwhile, the average driver is limited by its reaction speed limit and driving experience (generally the driver in the car when the slip angle of 2 degrees or less may be applied effectively manipulated, professional drivers, this angle will not exceed 4 degrees). Mutant vehicle dynamics will make it panic, unable to produce this sudden car skidding out of control effectively corrected.

\section{ESP system}

Currently wheel speed sensor uses electromagnetic induction two. In response to the low-speed electromagnetic induction type wheel speed sensor is relatively poor, and Hall sensors have better low-speed response. Steering wheel angle sensor is usually divided into optical encoders and potentiometers two [7, 8]. High accuracy optical encoder sensor, long life, but it is usually measured relative position, requiring zero point identification, and potentiometer can directly measure the absolute position.

Hydraulic actuator is the main executive body of the vehicle stability control system, the basic structure and ABS / ASR hydraulic actuator similar, in order to improve the response speed, vehicle stability control system pressure regulator than the ABS/ASR hydraulic modulator more pre pressure pump and the pressure generator. Fig. 1 is a schematic structure of HU5.0 hydraulic actuator. 


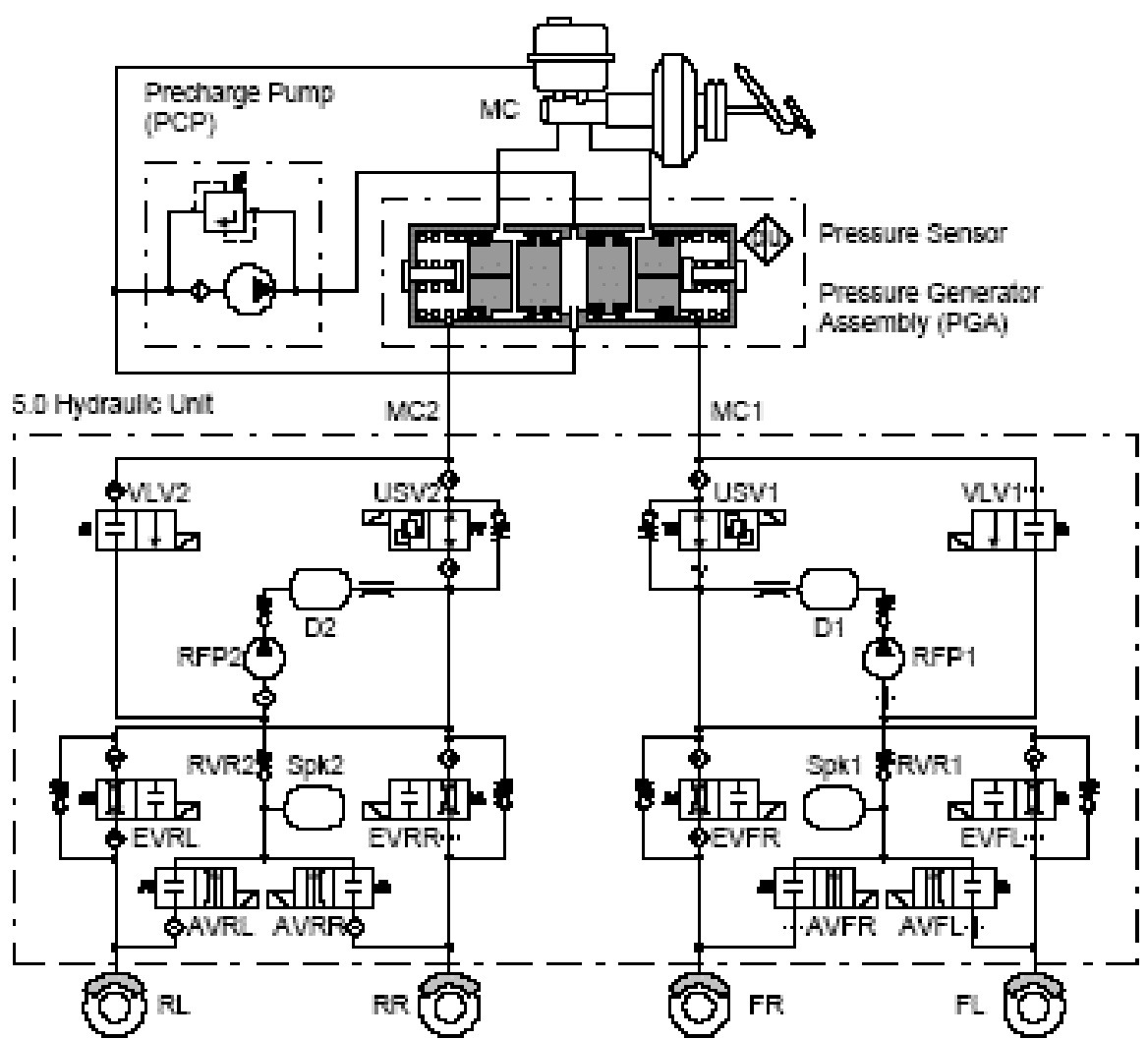

Figure 1. Schematic structure of HU5.0 hydraulic actuator

Vehicle stability control is a hot field of today's automotive active safety, compared with ABS, vehicle stability control has a great advantage, to improve vehicle safety in more complex conditions, which have been widely appreciated. Overseas, vehicle stability control in recent years has developed rapidly, and started a lot of equipment for medium and high-end cars. This article is in foreign research results, based on the study of the open-loop simulation and vehicles around the closed loop principle vehicle stability control, the control strategy of the main line system analysis.

\section{Characterization of the driver's intention of traveling}

Usually the driver is in the vehicle normal driving a car with state control. The so-called normal vehicle driving condition of a vehicle is very little traffic on the small curvature of the curve, there is no rapid overtaking, changing lanes when traveling state of emergency, and this time the vehicle lateral acceleration y is relatively small. In this case, the driver often based on pre-formed driving experience and understanding of the dynamics of the vehicle corresponding operation of the vehicle, and continue on the road traveling around the observation and reflection, were predictable (or front view preview) manipulate.

ESP system ideal yaw rate is calculated:

$$
\Psi_{N o 1}=\frac{v_{x} \cdot \delta_{W}}{(a+b) \cdot\left(1+\frac{v_{x}^{2}}{v_{C H}^{2}}\right)}
$$

The model takes into account the limits of adhesion, ideal between tire and road yaw rate reference value should be:

$$
\left|\Psi_{N o 1}\right| \leq \frac{\mu \cdot g}{v_{x}}
$$


Based on this: When you have a tendency to oversteer in a vehicle, the vehicle two degrees of freedom linear model can be used to describe the driver's intention with the ideal, but the vehicle's yaw rate does not increase, the linear model obtained by the two degrees of freedom ideal yaw rate is difficult to describe the ideal vehicle driving state. Resulting in this situation is because the two degrees of freedom model of the cornering force of the front axle has been restricted this failure condition of the two degrees of freedom model part will cause insufficient control algorithm is unable to determine the correct steering of the vehicle trend. Therefore, based on the low-pass filter characteristics of the vehicle, according to the researchers propose actual vehicle yaw rate inverse ideal driver steering wheel angle, compared with the actual steering wheel angle, in order to determine inadequate steering of the vehicle trend.

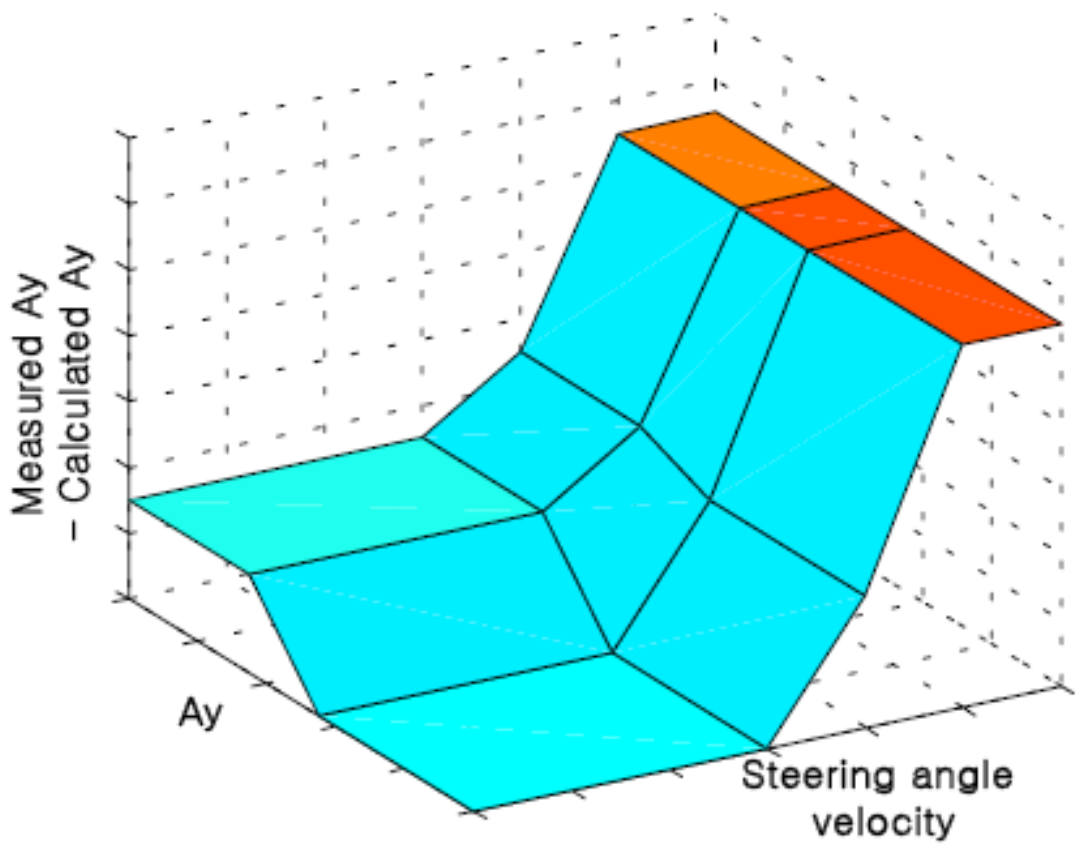

Figure 2. A lateral acceleration of the vehicle

Wherein, the driver steering wheel angle is calculated:

$$
\delta_{\text {calc }}=\frac{(a+b) \cdot\left(1+\frac{v_{x}^{2}}{v_{C H}^{2}}\right)}{v_{x}} \cdot \Psi
$$

Among them, the driver steering wheel angle is calculated: the state of the vehicle in extreme conditions generally are strongly nonlinear, a simple linear model has two degrees of freedom the monorail vehicle is difficult to accurately describe the vehicle motion characteristics in different driving conditions of the vehicle. ESP system for nonlinear control design, the establishment of a system for a vehicle reference model state based on the vehicle height interpolation table attached real vehicle test data, according to the vehicle steering wheel angle and vehicle speed difference obtained when different attachment steady road vehicle traveling from the interpolation table ideal yaw rate. In this case, the maximum yaw rate over the same restricted road friction coefficient. Fig. 2 is a lateral acceleration of the vehicle and the actual establishment of the goal of limiting the lateral acceleration difference threshold table.

Ideal yaw rate characterization is essentially linear region of the driver driving the mathematical characterization of experience compared to the linear model of two degrees of freedom. The aim is a more accurate characterization of the driver in different driving conditions driving intentions.

\section{Results and discussion}


Cars in the longitudinal acceleration and lateral acceleration under the effect of axle load transfer can be approximated by the following description.

Fig. 3 is a different road surface friction coefficient and longitudinal acceleration, vehicle sideslip angle of the steering wheel simulation curve characteristics. Each curve is left on the car sideslip angle generated when the front wheel steering vehicle yaw moment of impact; impact of car sideslip angle on the lateral force generated when the front wheel steering.
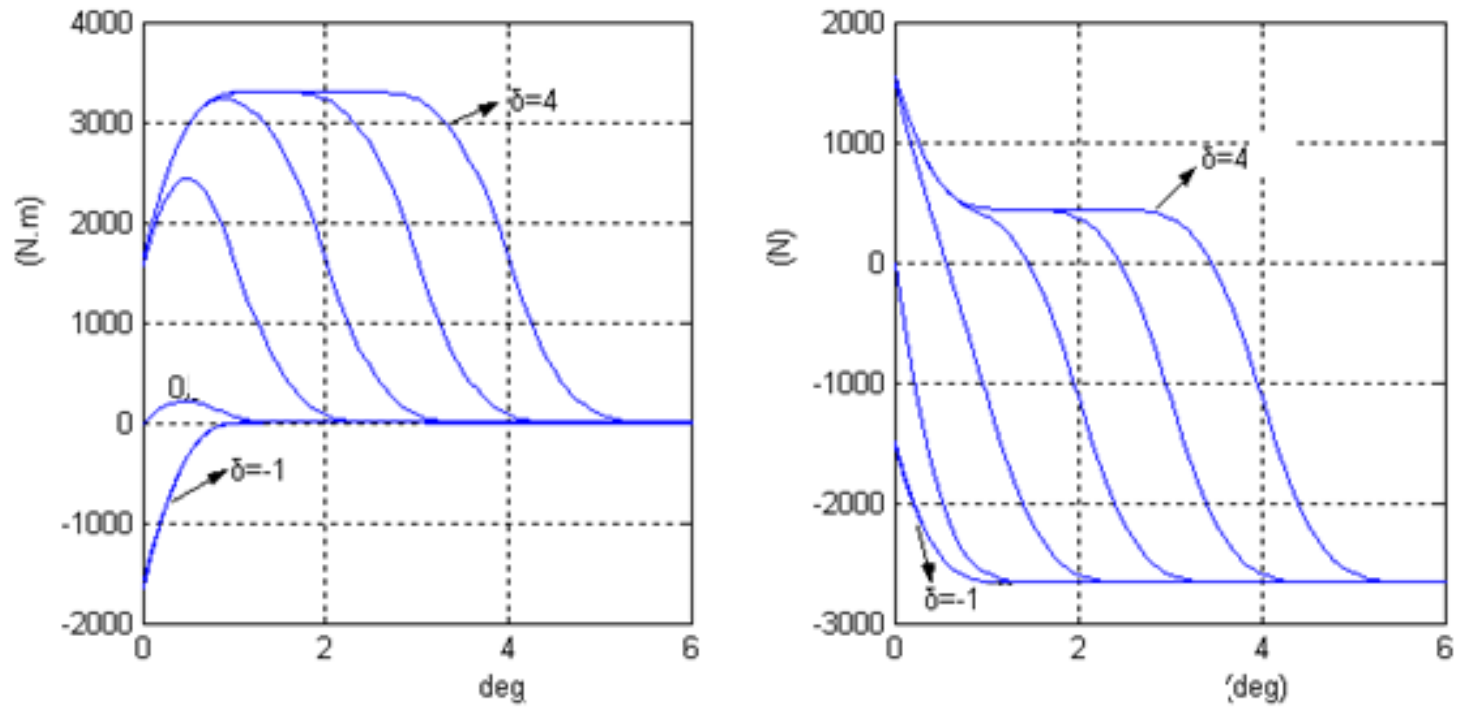

Figure 3. Live sub-module logical structure of teaching physical education under the module and implementation

\section{Summary}

Emerged on the market all kinds of stability control systems, with further reduction technology continues to progress and costs, vehicle stability control system has begun to equip middle and low cars. Due to the excellent performance of the vehicle stability control system, the system is likely to fully replace the ABS. ASR system has become the standard assembly of modern cars. Based on the research results at home and abroad on the basis of the main control strategy is investigated theoretically vehicle stability and provide a theoretical basis for future control system design. Vehicle stability control system of nonlinear time-varying characteristics, using a simple PID control method in consideration of the characteristics of the actuator based on the yaw rate and sideslip angle control strategies were studied.

\section{References}

[1] T.Ji and H.Shi, "Soft Sensor Modeling for Temperature Measurement of Texaco Gasifier Based on an Improved RBF Neural Network, " IEEE international Conference on Information Acquisition,pp.1147-1151,2006.

[2] T.Song, M.Jamshidi, R.R.Lee,et al., "A Novel Weighted Probabilistic Neural Network for MR Image Segmentation," IEEE International Conference on System,Man and Cybernetics, pp.2501-1506,2005.

[3] Multimedia information retrieval and management: Technological fundamentals and applications[M]. Springer Science \& Business Media, 2013.

[4] Y.L.Gao and Z.Z.Zhang, "Modulation Recognition Based on Combined Feature Parameter and Modified Probabilistic Neural Network," 6th World Congress on Intelligent Control and Automation, pp.2954-2958,2006.

[5] Chung C H, Pasquini L A, Koh C E. Web-based learning management system considerations for higher education[J]. Learning and Performance Quarterly, 2013, 1(4): 24-37. 
[6] Masayoshi Ito, Kenneth Yoshioka and Takeshi Saji, "Estimation of Road Surface Conditions Using Wheel Speed Behavior", International Symposium on Advanced Vehicle Control - AVEC’94, No.9438826

[7] Masugi Kaminaga, Genpei Naito, "Vehicle Body Slip Angle Estimation Using an Adaptive Observer”, International Symposium on Advanced Vehicle Control-AVEC’98, No.9836635.

[8] Ali Y. Ungoren, Huei Peng, H.E. Tseng, "Experimental Verification of Lateral Speed Estimation Methods”, International Symposium on Advanced Vehicle Control - AVEC 2002. 